\title{
Automated Content Analysis for Construction Safety: A Natural Language Processing System to Extract Precursors and Outcomes from Unstructured Injury Reports
}

\begin{abstract}
In the United States like in many other countries throughout the globe, construction workers are more likely to be injured on the job than workers in any other industry. This poor safety performance is responsible for huge human and financial losses and has motivated extensive research. Unfortunately, safety improvement in construction has decelerated in the last decade and traditional safety programs have reached saturation. Yet, major construction companies and federal agencies possess a wealth of empirical knowledge in the form of huge databases of digital construction injury reports. This knowledge could be used to better understand, predict, and prevent the occurrence of construction accidents. Unfortunately, due to the lack of a clear methodology and the high costs of manual large-scale content analysis, these valuable data have yet to be extracted and leveraged. Recently, researchers have proposed a framework allowing meaningful empirical data to be extracted from accident reports. However, the resource limitations inherent to manual content analysis still remain. The present study tested the proposition that manual content analysis of injury reports can be eliminated using natural language processing (NLP). This paper describes (1) the overall strategy and methodology used in developing the system, and specifically how key challenges with decoding unstructured reports were overcome; (2) how the system was built through an iterative process of coding and testing against manual content analysis results from a team of seven independent analysts; and (3) the implications and potential uses of the data extracted. The results indicate that the NLP system is capable of quickly and automatically scanning unstructured injury reports for 101 attributes and outcomes with over $95 \%$ accuracy. The main contribution of this research is to empower any organization to quickly obtain a large and highly reliable structured attribute and outcome data set from their databases of unstructured accident reports. Such structured data are a necessary prerequisite to the application of statistical modeling techniques allowing the extraction of new safety knowledge and finally the amelioration of safety management.
\end{abstract}

\section{MOTIVATION}

Construction is constantly ranked as one of the most dangerous industries worldwide (Sacks et al. 2009). In the United States, despite the improvements that followed the Occupational Health and Safety Act of 1970, construction still accounts for $17 \%$ of all work-related deaths while only employing $7 \%$ of the national workforce (CPWR 2013). In fact, according to the Bureau of Labor Statistics (2014), approximately 700 workers die each year. Construction fatalities and injuries result in immense societal costs, totaling approximately $\$ 15$ billion in

Page 1 of 24 
lost revenue every year (BLS 2011). What is even more alarming is that these colossal human and financial costs are expected to escalate with the 33\% construction employment growth projections in the 2010-2020 decade, which is more than twice the overall anticipated economic growth (CPWR 2013).

Despite the abundant research that has been motivated by the aforementioned alarming injury and fatality rates, safety performance in construction has been plateauing in recent years and the implementation of effective injury prevention practices has reached saturation (Esmaeili and Hallowell 2012). Fortunately, risk-based approaches are emerging and show promise for safety improvement through proactive decision-making. For example, Baradan and Usmen (2006) compared the risk of building trades, Hallowell and Gambatese (2009) quantified the safety risk for various activities required to construct concrete formwork, and Shapira and Lyachin (2009) studied the impact of tower cranes on jobsite safety. However, these approaches are currently limited because (1) they focus on specific activities and trades without considering the temporal and spatial interactions among risk factors; (2) they are not based on empirical data; and (3) they are limited in scope of application (Prades 2014, Sacks et al. 2009). Consequently, existing models do not translate well to other work scenarios, and do not capture the dynamics of construction work, where trades and activities constantly interact (Sacks et al. 2009, Helander 1991). To overcome these limitations, Esmaeili and Hallowell (2012) proposed a unified attribute-based framework that allows standard risk factor and outcome variables to be extracted from naturally occurring accident reports. Although this method shows promise, it requires the analysis of large numbers of reports to see patterns and trends emerge from the data. Such manual content analysis is laborious and resource-intensive (Prades 2014, Desvignes 2014).

To remove the needs for manual analysis of injury reports and allow the large-scale use of the attribute-based framework, the present study tests the proposition that attributes and safety outcomes can be automatically and accurately extracted from unstructured injury reports using natural language processing (NLP).

\subsection{BACKGROUND: ATTRIBUTE-BASED APPROACH TO CONSTRUCTION SAFETY}

The attribute-based approach to construction safety theorizes that any construction situation can be uniquely and comprehensively characterized by a finite number of observable fundamental construction site attributes (Prades 2014; Desvignes 2014). These basic elements are context-free, universal, and pertain to construction means and methods, environmental conditions, and human factors. For instance, in the following excerpt of an injury report: "employee was welding overhead and wind shifted, resulting in discomfort to left eye", three fundamental attributes can be identified: (1) welding, (2) working overhead, and (3) wind.

Although this approach is simple, it is very powerful. First, from this perspective, any incident can be viewed as the resulting outcome of the presence of a worker and the joint occurrence of some fundamental attributes. Consequently, attributes are also called injury precursors, or simply precursors. In what follows, the terms attribute and precursor are used interchangeably. It is important to note that precursors should be observable before an injury occurs. Falling object, for example, is not a precursor, it is an outcome. On the other hand, object at height is a precursor. As illustrated in the previous example, descriptors of the work environment and outcomes can be extracted even from brief reports. Finally, this information is authentic since it is simply based on objective narratives of discrete events.

A connection with genetics can naturally be made with this style of analysis: every person is unique, but their genetic information is entirely encoded by combinations of a finite number of basic universal building blocks that constitute their DNA. The attribute-based approach to construction safety is built upon a similar theory that by identifying fundamental and universal construction injury precursors, understanding how they interact, and modeling how they shape risk and create unsafe work conditions, it may be possible to better understand the true nature of, predict, and prevent the occurrence of construction injuries. Historically, scientific understanding of complex phenomena has improved when breaking down convoluted systems into fundamental constituents that individually are easier to comprehend. A fascinating recent example is the Human Genome Project (Collins et al. 2003), which allowed sequencing and mapping of about 30,000 genes, unlocking the structure of human DNA. Similarly, the finite element method, a numerical technique used in many quantitative disciplines of engineering, 
is built on the theory that complicated continuous structures and objects can be represented by a finite number of geometrically simpler pieces (Zienkiewicz et al. 1971).

Esmaeili and Hallowell (2012) conducted the first attribute-level risk analysis in construction by analyzing 300 struck-by injury cases from national databases. Through this analysis, they identified 34 fundamental attributes. More recently, a team of eight researchers performed a manual content analysis of 2,201 industrial injury reports gathered from 476 contractors, allowing the initial list of Esmaeili and Hallowell (2012) to be refined and broadened to 80 precursors (Prades 2014, Desvignes 2014). These precursors are summarized in Table 1. The validity of the content analysis and relevance of the attributes presented by Prades (2014) and Desvignes (2014) was ensured by adhering to a strict coding scheme, implementing an iterative process with team-based calibration meetings, and using peer reviews and random checks by external reviewers. In these studies, attributes were classified in three categories: upstream, transitional, and downstream. Upstream precursors can be anticipated as soon as during the design phase; transitional precursors are generally not identifiable by designers but can be detected before construction begins based on knowledge of construction means and methods; and downstream precursors are mostly related to human behavior and can only be observed during the construction phase.

Table 1. Context-free validated injury precursors from Desvignes (2014)

\begin{tabular}{|c|c|c|}
\hline UPSTREAM & Rebar & Screw \\
\hline Cable tray & Scaffold & Slag \\
\hline Cable & Soffit & Spark \\
\hline Chipping & Spool & Slippery walking surface \\
\hline Concrete liquid & Stairs & Small particle \\
\hline Concrete & Steel sections & Adverse low temperatures \\
\hline Conduit & Stripping & Unpowered tool \\
\hline Confined workspace & Tank & Unstable support/surface \\
\hline Congested workspace & Unpowered transporter & Wind \\
\hline Crane & Valve & Wrench \\
\hline Door & Welding & Lifting/pulling/manual handling \\
\hline Dunnage & Wire & Light vehicle \\
\hline Electricity & Working at height & Exiting/transitioning \\
\hline Formwork & Working below elevated workspace/material & Sharp edge \\
\hline Grinding & Drill & Splinter/sliver \\
\hline Grout & TRANSITIONAL & Repetitive motion \\
\hline Guardrail/handrail & Bolt & Working overhead \\
\hline Heat source & Cleaning & DOWNSTREAM \\
\hline Heavy material/tool & Forklift & Improper body position \\
\hline Heavy vehicle & Hammer & Improper procedure/inattention \\
\hline Job trailer & Hand size pieces & Improper security of materials \\
\hline Lumber & Hazardous substance & Improper security of tools \\
\hline Machinery & Hose & No/improper PPE \\
\hline Manlift & Insect & Object on the floor \\
\hline Stud & Ladder & Poor housekeeping \\
\hline Object at height & Mud & Poor visibility \\
\hline Piping & Nail & Uneven walking surface \\
\hline Pontoon & Powered tool & \\
\hline
\end{tabular}

121

122

123

124

125

126

127

128

129

130

In addition to the 80 precursors presented in Table 1, Prades (2014) and Desvignes (2014) also extracted various safety outcomes from accident reports, namely injury type, injury severity, body part affected, and energy source involved. The variables belonging to these categories are listed in Table 2 . The injury codes, severity levels, and body divisions included in Table 2 are consistent with OSHA definitions and past research (Hallowell 2008). Energy sources were extracted based on the theory that any injury can be associated with the release of an energy source (Fleming 2009, Haddon 1973). For instance, a falling load can be seen as a gravity release, a welding flash burn involves radiation, and waterproofing substances, solvents, or concrete in its liquid form can cause chemical burns. Additional definitions and examples can be found in Albert et al. (2014). Attributes and outcomes are occasionally referred to as variables in the rest of this paper. 
131

132

133

Table 2. Outcome categories from Prades (2014) and Desvignes (2014)

\begin{tabular}{llll} 
INJURY CODE & INJURY SEVERITY & $\begin{array}{l}\text { BODY PART } \\
\text { AFFECTED }\end{array}$ & $\begin{array}{l}\text { ENERGY SOURCE } \\
\text { INVOLVED }\end{array}$ \\
\hline Caught in or compressed & Pain & Head & Biological \\
Exposure to harmful substance & First aid & Neck & Chemical \\
Fall on same level & Medical case & Trunk & Electricity \\
Fall to lower level & Lost work time & Upper extremities & Gravity \\
Overexertion & Permanent disablement & Lower extremities & Mechanical \\
Struck by or against & Fatality & & Motion \\
Transportation accident & & & Pressure \\
& & & Radiation \\
& & & Thermal
\end{tabular}

Although the work of Esmaeili and Hallowell (2012), Prades (2014), and Desvignes (2014) made important contributions to attribute-level safety analysis, the manual content analysis procedures used were time consuming, limiting the number of reports that could be analyzed in a reasonable research effort, and thereby the emergence of trends and patterns in the data extracted. For example, Desvignes (2014) only used a random set of 1,280 reports from a larger set of 4,458 available reports because of time and resource limitations. In addition, even when a rigorous protocol is followed, it is never possible to entirely eliminate inconsistencies among human coders. For all these reasons, resorting to manual content analysis to systematically mine large databases of construction injury reports is not viable. In order to eliminate the needs for manual analysis of reports, and allow very large databases to be leveraged, this study introduces a fully automated and highly accurate NLP system.

In construction safety research, the only known attempt of automatically analyzing injury reports was made by Esmaeili (2012). In this study, high severity injury reports contained in national databases were scanned for 22 attributes with the NVivo (QSR International 2011) commercial software. Though this effort involved extracting attributes from injury reports for the first time, it suffers some limitations. First, the reliability of the attribute identification and keyword validation process is questionable because fewer than 500 accident reports were used to identify attributes and tune keywords, and a percent agreement score of 0.7 was set as the accuracy threshold. This is a rather low value, especially since percent agreement is known to be a lenient metric that inflates agreement in all cases (Lombard et al. 2002, Iacobucci and Dawn 2001). Second, the validated list of keywords, and explanations about how NVivo was used to automate the content analysis were not provided, making replication of the work and assessment of the quality of the data obtained impossible. Third, only high severity struck-by injuries were studied, which significantly narrowed the breadth of attributes that could be identified and the quantity, relevance and generalizability of the keywords found. Fourth, some precursors were defined in opposition with the conceptual attribute-based framework. For instance, falling object, structure collapse, or falling out from heavy equipment cannot be considered injury precursors, because they are outcomes rather than observable characteristics of the jobsite. This paper seeks to collectively address these limitations.

\subsection{BACKGROUND: NATURAL LANGUAGE PROCESSING}

Natural language processing (NLP) is a very active and rapidly evolving area of research that deals with the comprehension and analysis of human-produced texts by computers (Chowdhury 2003). It enables machines to derive meaning from human language input. NLP lies at the confluence of artificial intelligence, linguistics, and computer science, and aims at achieving human-like natural language understanding (Liddy 2001). Applications of NLP include speech recognition, machine translation, and automated content analysis (Manning and Schuetze 1999).

Automated content analysis is increasingly being used for a variety of applications. This can be explained by the needs to make sense of and leverage the fast-growing volume of digital information (Bai 2011). In construction, even a small project generates a lot of electronic information in the form of specifications, computer aided drawings, process control, inventory management, cost estimating, scheduling, and other documentation 
(Soibelman et al. 2008). For many years, major companies and federal agencies have also been constituting extensive databases of electronic injury and near miss reports as part of their safety programs.

In a recent study, Francis and Flynn (2010) attempted to categorize insurance claim descriptions into four categories based on keywords, and then used the clustering to predict claim severity. For instance, the claims corresponding to car accidents were automatically extracted based on the keywords hit, travel and vehicle. In another study, 10,000 traffic incident reports were automatically categorized into topics using Latent Dirichlet Allocation and incorporated into predictive models to forecast time-to-clearance and improve traffic management in real time (Pereira et al. 2013). In the construction industry, text analytics have been used to classify project documents (Al Qady and Kandil 2014, Caldas and Soibelman 2003), to retrieve computer aided drawings from databases (Yu and Hsu 2013), to automate knowledge extraction from narratives and represent it as a map (Yeung et al. 2014), and to structure and manage safety knowledge in order to support Job Hazard Analysis (Chi et al. 2014, Wang and Boukamp 2011).

Creating and validating a system that understands natural language is very challenging. Natural languages are complex, consisting of a catalogue of words, called a lexicon, and set of structural rules, called a grammar, that allows meaning to be built by combining words into sentences (Manning and Schuetze 1999). Historically, the most serious obstacle to effectively analyze naturally occurring language has been the difficulty to accurately model grammars (Hindle 1989). Additionally, many words have several meanings, making the use of word sense disambiguation indispensable.

Most modern natural language processing tools use machine learning algorithms and statistical modeling to overcome the aforementioned barriers. Some of the most widely used techniques in text analytics are clustering algorithms, such as k-means, and classification algorithms, such as k-nearest neighbors, naïve Bayes, and Support Vector Machines (Grimmer and Stewart 2013, Verma et al. 2011, Li and Desheng 2010, Prabowo and Thelwall 2009, Karatzoglou et al. 2010, Manning and Schuetze 1999). Some popular methods also include Random Forest (Grimmer and Stewart 2013), graph theory (Ferrer i Cancho and Sole 2001), Bayesian and Markov models (Bai 2011), and Latent Dirichlet Allocation (Pereira et al. 2013).

\section{SELECTION OF AN APPROPRIATE NATURAL LANGUAGE PROCESSING METHOD}

In developing the automated content analysis tool, a dilemma arose. Ideally, machine learning algorithms would have been applied to the available data manually-coded by Prades (2014) and Desvignes (2014). Unfortunately, these techniques, such as Support Vector Machines, perform poorly when a sufficient number of positive training examples is not available for each category (Prabowo and Thelwall 2009). Some researchers have suggested that to attain effective statistical learning, 75 to 100 positive cases per category is an absolute minimum (Beleites et al. 2013, Hopkins and King 2010). Due to the high dimension of the injury report feature space (80 attributes) and the diversity of construction situations, the available training data were naturally sparse. For instance, in the report "carpenter felt discomfort in his left knee while exiting a tight area", only 2 attributes are present, namely exiting/transitioning and confined workspace. The other 78 attributes are not featured. Similarly, only a couple of attributes co-occur in each injury report. Therefore, for a large number of reports, cases when a given attribute is present are outnumbered by cases when this same attribute is not present. For example, in Desvignes' (2014) dataset of 1,280 manually-analyzed reports, the median attribute in terms of number of appearances, heavy vehicle, occurs only 21 times. Therefore, manually analyzing tens of thousands of injury reports would have been required to put together a satisfactory training database and achieve efficient machine learning.

Because such a large number of reports was not available, and because of time and resource limitations, the team decided to design a NLP program based on hand-coded rules and dictionaries of keywords. Though this approach is not as simple and elegant, it offers advantages over machine learning. Most importantly, it allows researchers to integrate human intelligence and knowledge of the data into the system, allowing higher levels of accuracy to be reached (Sagae and Lavie 2003). As Wang et al. (2002) described, statistical classifiers are targeted at broad and relatively shallow understanding, whereas hand-crafted rules perform well within a specific domain when deep 
understanding is sought. Changes in coding scheme, detection of new variables, and higher skill can also be achieved very quickly by simply updating the rules and dictionaries, whereas algorithms require new, expensive training data to get better. Additionally, rule-based tools avoid the relatively opaque nature of machine learning (Barbella et al. 2009, Breiman 2001).

\section{DESIGN OF A RULE-BASED AUTOMATED CONTENT ANALYSIS SYSTEM}

The R programming language ( $\mathrm{R}$ Core Team 2014) was used to develop a fully automated NLP system based on hand coded rules and dictionaries of keywords. Although there are other languages that could have been used, $R$ was preferred because it is open source, increasingly being used, and features high quality libraries to more efficiently build on the previous work of others. The coding scheme, operational definitions of variables, and knowledge gained from the manual content analysis of more than 2,200 injury reports (Prades 2014, Desvignes 2014) were used to design the tool. Examples of injury reports from Desvignes (2014)'s database are provided in Table 3. Although these reports are not lengthy, they were written by personnel working on site within 8 hours of the injury as requested by the participating contractors' policies, and contain enough details to have a good idea of the work environment at the time of the accident and the injury outcomes. Other report examples can be found in Table 5 .

Table 3. Examples of injury reports from Desvignes et al. (2014), with attributes and outcomes

\begin{tabular}{|c|c|c|c|c|}
\hline Reports & Attributes & Energy source & Injury code & Body part \\
\hline $\begin{array}{l}\text { "A finisher apprentice was applying crystalline } \\
\text { waterproofing to construction joints and cracks inside a } \\
\text { pontoon cell. At some point the waterproofing material } \\
\text { got in-between his kneepad and wet jeans which caused a } \\
\text { concrete burn on his leg. The area was treated with a } \\
\text { burn spray and he employee returned back to work } \\
\text { immediately." }\end{array}$ & $\begin{array}{l}\text { Hazardous substance, } \\
\text { pontoon }\end{array}$ & Chemical & $\begin{array}{l}\text { Exposure } \\
\text { to harmful } \\
\text { substance }\end{array}$ & $\begin{array}{l}\text { Lower } \\
\text { extremities }\end{array}$ \\
\hline $\begin{array}{l}\text { "As employee was walking, he stepped on a nail that was } \\
\text { lying in the road base/gravel. The nail was lying free on } \\
\text { the ground. Employee felt the nail puncture his foot and } \\
\text { immediately pulled it out and reported it to the safety } \\
\text { representative who was nearby." }\end{array}$ & $\begin{array}{l}\text { Nail, uneven walking } \\
\text { surface, object on the } \\
\text { floor }\end{array}$ & Motion & $\begin{array}{l}\text { Struck by } \\
\text { or against }\end{array}$ & $\begin{array}{l}\text { Lower } \\
\text { extremities }\end{array}$ \\
\hline $\begin{array}{l}\text { "Employee was lifting a } 2 \times 12 \text { wood plank when the } \\
\text { wood plank got too heavy causing it to fall back towards } \\
\text { the employee and hit him on the top/front of his hard } \\
\text { hat." }\end{array}$ & $\begin{array}{l}\text { Lumber, heavy material, } \\
\text { lifting/pulling, improper } \\
\text { security of materials }\end{array}$ & Gravity & $\begin{array}{l}\text { Struck by } \\
\text { or against }\end{array}$ & Head \\
\hline $\begin{array}{l}\text { "At the ABC site wet tailrace a worker went to the Sea- } \\
\text { Can for tools and PPE. When he went to open the door of } \\
\text { the Sea-Can he received a } 120 \text { volt shock." }\end{array}$ & Door, electricity & Electricity & $\begin{array}{l}\text { Exposure } \\
\text { to harmful } \\
\text { substance }\end{array}$ & $\begin{array}{l}\text { Not } \\
\text { detectable }\end{array}$ \\
\hline $\begin{array}{l}\text { "A pipefitter was welding on a pipe support when slag } \\
\text { fell into the cuff of his left glove, resulting in a burn to } \\
\text { his left wrist." }\end{array}$ & $\begin{array}{l}\text { Welding, steel sections, } \\
\text { slag }\end{array}$ & Thermal & $\begin{array}{l}\text { Exposure } \\
\text { to harmful } \\
\text { substance }\end{array}$ & $\begin{array}{l}\text { Upper } \\
\text { extremities }\end{array}$ \\
\hline
\end{tabular}

The system was built to automatically and accurately scan injury reports for the 80 validated attributes, 7 injury types, 5 body parts, and 9 energy sources summarized in Tables 1 and 2. Some minor differences with Desvignes' (2014) original classification are to be noted: object at height on same story was grouped with the attribute object at height, and the precursors slag and sparks were separated. The attribute snow/ice was extended to include low temperature incidents (e.g., cases of hypothermia) and was renamed adverse low temperatures. The attributes unpowered hand tool and powered hand tool were extended to include tools that are not hand-held, but do not belong either to the category machinery (e.g., girder spacer, power trowel, etc.). Therefore, these attributes were renamed powered tool and unpowered tool. Also, one attribute, improper procedure/inattention, was added. Finally, the injury types struck by and struck against were grouped under the umbrella struck by or against, which 
is consistent with the Occupational Injury and Illness Classification System (OIICS) and BLS classifications (Hallowell 2008).

As shown in Figure 1 and as previously mentioned, NLP systems generally consist of a lexicon, or catalogue of words, and a set of structural rules, called a grammar, allowing meaning to be derived from the way words are combined (Manning and Schuetze 1999). Figure 2 describes the tool building and validation process. In what follows, details about the construction of the lexicon and grammar are given.

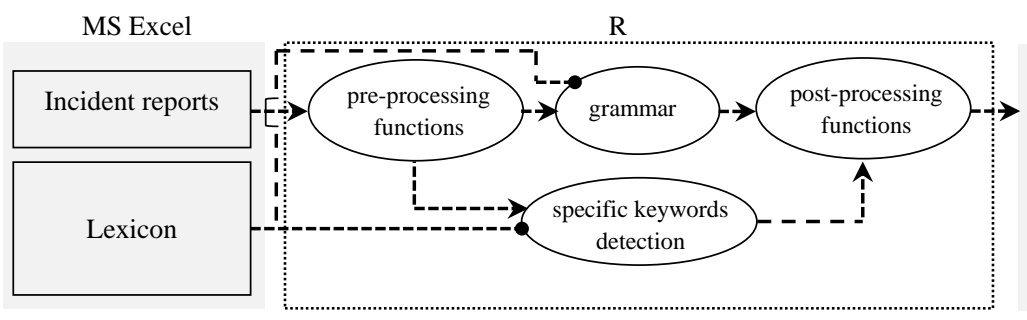

MS Excel

Figure 1. Overarching NLP tool process flow

Attributes, energy source, injury code and body part for each report



Figure 2. NLP tool building and validation process

\subsection{Step 1: lexicon building}

The first major step in the design of the automated content analysis system was the development of keyword dictionaries. Although there has been a great deal of construction safety research, no lexicon related to precursors of construction injuries was available at the time of this research. To create such a lexicon, several resources were leveraged as shown in Figure 2 and as discussed below. 
The first resource for this inquiry was the 2,201 manually analyzed incident reports from Prades (2014) and Desvignes (2014). These data include the attributes and outcomes shown in Tables 1 and 2 for each report, thereby allowing systematic sorting and identification of common keywords and phrases linked to each attribute. Online resources, such as the OSHA website, were subsequently used for dictionary enrichment. The focus of this section of the paper is on this decomposition process and the creation of the lexicon.

\subsubsection{Specific keywords}

In order to initiate keyword dictionary creation, the R "tm" package (Feinerer and Hornik 2014) was used. The hand-analyzed reports were sorted by attributes and outcomes and the most frequent terms associated with each group of reports were found. For example, some of the most frequent words associated with the energy source chemical were "burn", "irritation", "line", "liquid", "concrete", "water", "chemical", "caustic", "eye", "acid", "cloud", "face", “coker", "laborer", "burning", "skin", "insulation", “insulator", "mist", "splatter", "waterproofing", "sprayed", and "flushed." Although only a simple frequency count, this list is very insightful. A first observation can be made that some of the keywords in the list, such as "caustic", "acid", and "chemical", are very specific to the energy source chemical. Indeed, the sole occurrence of these terms in a given incident report would suffice to classify the report as a chemical injury. Single keywords are known as unigrams (Manning and Schuetze 1999), and "caustic", "acid", and "chemical", can thus be considered to be unigrams specific to the energy source chemical.

Within the context of chemical energy, the keyword "concrete" is an excellent example where additional words were required to derive meaning and to properly identify when concrete-related incidents were related to the chemical properties of the material. For instance, "concrete pouring" denotes a task, "concrete bag" a heavy material, "concrete drill" a powered tool, "concrete blanket" a type of tarpaulin, "concrete foreman" a person, and "concrete burn" refers to a type of chemical burn. Accordingly, although the sole presence of "concrete" or "burn" does not allow the variable chemical to be detected (i.e., "concrete" and "burn" are not unigrams specific to chemical), when these two words are found in a report as a pair (i.e., "concrete burn"), there is no ambiguity that the report deals with a chemical incident. Thus, "concrete burn" can be considered to be a specific double keyword (i.e., a specific bigram), associated with the energy source chemical.

The most frequent terms for all variables were carefully inspected, and unigrams, bigrams, and trigrams specific to each of the 101 variables (i.e., 80 attributes, 9 energy sources, 7 injury types, and 5 body parts) were gathered. These terms were then arranged in a lexicon composed of one catalogue of terms, or dictionary, for each variable. Online resources allowed the dictionaries to be enriched in order to anticipate new cases. For instance, synonyms of "caustic", such as "corrosive", "irritative", "toxic", etc., can be looked up and added to the dictionary for chemical even if these particular keywords are not found directly in the reports. Other online resources such as the OSHA website also contain a tremendous amount of valuable keywords and were used to augment the dictionaries even more. Most of the keywords found in the final dictionaries were gathered by adopting this "generalization and anticipation" process as shown in Figure 2. Some examples of these dictionaries of specific keywords include steel sections (37 unigrams, 80 bigrams, 2 trigrams), lumber (46 unigrams, 34 bigrams, 26 trigrams) and poor housekeeping (4 unigrams, 3 bigrams). Although specific keywords allow easy variable detection in some cases, they are not sufficient for detecting all variables in all cases and it is necessary to look at generic keyword combinations to decipher meaning from text.

\subsubsection{Generic keywords}

In many cases, variables have to be detected based on combinations of keywords that are not specific to any variable. These keywords are referred to as generic keywords. Looking closely at the list of keywords for the energy source chemical, one can note that some keywords, such as "insulator", "laborer", "eye", or "skin", are related to persons, while some others refer to actions ("flushed", "sprayed"), materials ("insulation"), outcomes ("irritation"), or location (a "coker" is an oil refinery unit). None of these keywords alone, if found in an injury 
report, would guarantee the presence of the variable chemical, nor of any other variable. However, chemical should be detected if "insulation" is associated with "eye", "skin", "irritation", or "burning". Therefore, all the keywords related to the idea of irritation (e.g., "irritation", "itching", "burning", etc.), all the keywords dealing with the human body (e.g., "skin", "eye", "hand", etc.), and all the keywords about insulation (e.g., "insulation", "fiberglass", "glass wool", "foam", etc.) were collected and stored in dedicated dictionaries (adopting the anticipation and generalization process previously discussed), and a grammatical rule for the detection of the energy source chemical was created. Another example when an attribute can be identified based on generic keywords is working at height. This precursor should be detected when a term linked to the topic of working (e.g., "working", "doing", "performing", "drilling", "installing", etc.) is associated with a keyword involving the notion of height (e.g., "height", "elevation", "elevated", "mezzanine", "roof”, etc.). These simple examples are provided to illustrate the spirit of the variable detection strategy, but in practice, relying simply on association is not enough to avoid false alarms. Detection rules (also called grammatical rules) have to take into account the order in which the keywords appear, and context. The rule building process will be described in detail in the next section.

Since generic keywords do not allow specific variables to be detected until they are combined with other keywords, generic keywords were not organized in variable-related dictionaries, but rather, stored in topic or concept-related dictionaries. Some of these 47 dictionaries are, for example, people (141 unigrams, 8 bigrams, plus the 475 most given first names in the U.S.), working (117 unigrams, 1 bigram), elevation (21 unigrams, 1 bigram, 1 trigrams), and unstable (47 unigrams, 24 bigrams).

The notions of specific and generic words originate from the field of linguistics. For example, Ferrer I Cancho and Sole (2001) observed, using graph theory, that there are two different regimes of words: basic and specialized. Also, it is important to keep in mind that dictionary creation is an iterative process. No lexicon is fully comprehensive and careful inspection of the system's errors, deep understanding of the textual data, and full use of available linguistics resources are required to ensure a continuous improvement of the dictionaries (Grimmer and Stewart 2013). Such updating, while time-consuming and sometimes tedious, is necessary (Kuechler 2007). The most recent version of the lexicon that was developed for this research is available on request by emailing the corresponding author. As shown in Figure 2, and as will be discussed, errors were closely examined during the validation process, and keywords and rules were tuned accordingly.

\subsection{Step 2: Devising detection rules}

The second step in the development of the system was the writing of grammatical rules to detect variables based on combinations of generic keywords in the text. Detecting variables using generic keywords is complex. Simply testing for co-occurrence of topics creates many errors. To illustrate, consider the following two injury report excerpts:

Excerpt 1: "moving floor plate, employee strained his back."

Excerpt 2: "employee rolled his ankle on moving floor plate."

In each excerpt, the generic keywords in bold are associated with the same topics and appear in the exact same order. Yet, very different precursors should be identified in each case. In the first report, it should be inferred that a back injury was sustained as a result of the presence of the attribute material handling, whereas in the second report, the worker sprained their ankle due to the occurrence of the precursor unstable support/surface. The ambiguity comes from the fact that the bigram "floor plate" has two different senses: (1) material and (2) walking surface. If "floor plate" refers to some material, then it is being moved (necessarily by a person) and "moving" is a verb. On the other hand, if "floor plate" designates a walking surface, then it is moving as a result of an unintended action or some exterior influence and "moving" is used as an adjective to qualify "floor plate".

To detect the correct attribute, it is necessary to determine which of the senses of "floor plate" is invoked in each report, or in other words, to disambiguate the sense of the generic bigram "floor plate". In many instances, disambiguation is helped by looking at the context, such as looking at preceding and following words. Also, 
taking into account prepositions such as "on", “onto", “into", "under", and "over" gives a lot of information. Finally, the structure of the injury reports can be decomposed by using punctuation marks such as commas and periods, and conjunctions such as "and", "or", and "while". In the given example, it should be noted that the attribute unstable support/surface can be rightfully identified in the second report if the fact that the preposition "on" precedes "floor plate" is captured.

26 variables were detected based on their specific keywords only. For the energy source biological, for instance, the occurrence of "biosludge", "scorpion", "bees", "bugs", or "wastewater" (and others very specific, unambiguous keywords) was enough to trigger detection. However, for 76 of the 101 variables, it was necessary to account for the subtleties previously mentioned, namely (1) neighboring generic or specific keywords (context), (2) report structure (punctuation, conjunctions), and (3) ordering of terms. As shown in Figure 2, the signature of each of these variables was empirically determined from knowledge gained during the manual content analysis of more than 2,200 reports, from generalization, anticipation, and grammatical logic, and from lessons learned during the tool tuning process. These signatures were transposed into the $\mathrm{R}$ programming language in the form of hand-crafted detection rules.

Within the 76 variables requiring grammatical rules, 51 were detected based on a combination of specific keywords and rules, and 25 did not have specific keywords and were detected based on rules alone. An example of an attribute with mixed rules is confined workspace, where "potholing", "manhole", "tunnel", and others were used as specific keywords. To capture all other cases, the variable was detected if any element from the generic family confined (e.g., "confined", "limited", "tight", "narrow", etc.) was found combined with any element from the generic family area (e.g., "area", "room", "space", "quarters", "entrance”, etc.).

The remaining 25 variables were detected based on rules alone. For instance, the grammatical rule for the attribute object at height can be written in English as "(any element from the topic materials.tools is followed by any element from the topic fall) AND (no element from the topic people is found sandwiched between any element from the topic materials.tools and any element from the topic fall) AND (any element from the topic elevation is present). For cable, the rule is much simpler, and can be written in English as "any of the keywords ("cable", "cables") is present, but is not immediately followed by any of the keywords ("tray", "shovel", "wheel", "reel", "spool", "coil", or "trench")". Indeed, cable tray is another attribute, a cable shovel is classified as a heavy vehicle, wheels, reels, coils and spools are classified in the attribute spool, and "cable trench" is not specific to cable (e.g., "carpenter tripped on cable trench"). Note that this is done without loss of generality, since both "cable" and "cable trench" are free to co-occur. In the sentence "worker was installing cable in cable trench", cable would be detected.

To efficiently write these statements in the $\mathrm{R}$ programming language, a library of custom functions was developed.

\subsubsection{Creation of $R$ functions}

Table 4 shows the comprehensive list of functions that were created to write the rules. Every single rule was written as a combination of statements involving these functions. Before being passed to these functions, as shown in Figure 1, unstructured text needs to be cleaned and converted into structured data. Functions from the "base" (R core team 2014) and "tm" (Feinerer and Hornik 2014) R packages were used to perform these preprocessing steps.

Preprocessing included (1) converting the text to lower case ( $\mathrm{R}$ is case sensitive), (2) removing some punctuation marks, (3) eliminating words containing little or no information, (known as stopwords) and finally (4) stripping the extra white space. In removing punctuation (step 2), commas and periods were kept, since they provide valuable information about text structure. For example, in: "accident involved one welder. Falling hammer from mezz deck struck him", the immediate proximity between the two keywords "welder" and "falling" should be disregarded because these two words do not belong to the same sentence. Hence, the machine should understand 
that the welder himself is not falling. The third step, stopwords removal, is standard and used to some degree in all text mining applications (e.g., Pereira et al. 2013, Caldas and Soibelman 2003). Stopwords such as "what", "too", "a", "be" and others were removed, but words referring to persons, such as "she", "he", "they", "his", "her", etc. as well as other words like some prepositions and conjunctions (e.g., "below", "between", "into", "and", "so", etc.), proved useful and were kept. Numbers and intra-word dashes were also kept, since a number of keywords include such characters. For instance, some specific unigrams for the attribute lumber are "2x2", "2x6", and some for the precursor bolt are "she-bolt" and "u-bolt". Finally, the last step consisted in splitting the text based on whitespace. This action turns unstructured text into an ordered character vector. The elements of the vector can be words, punctuation marks (commas and periods), and numbers. Each element is automatically assigned an index number, which corresponds to the position of the element in the ordered vector of words (i.e., in the text). This step is fundamental, as it allows distance between elements, called "radius" in what follows, to be measured.

The key assumption when working with radii is that only words close to each other are related. Beyond a certain distance, words are not connected anymore. This is consistent with the Markov assumption in natural language processing, which holds the local context of a word only to be of importance (Manning and Schuetze 1999). In time series analysis, an equivalent approach is used: the correlation between observations decreases and eventually disappears as temporal distance increases (Wei 1994, pp.6-26).

\section{Table 4. Comprehensive list of functions used for writing rules}

\section{FUNCTION}

statement1 | statement2

statement $1 \&$ statement 2

any $(\mathrm{a} \%$ in\% $\mathrm{x})$

any(sapply(a, grepl, text))

complex.s(a, b, radius, text)

tricky.double(a, b, c, number, radius, text)

\section{RETURNS TRUE IF... (FALSE ELSE)}

(statement 1 is true) or (statement 2 is true)

(statement 1 is true) and (statement 2 is true)

any unigram of the character vector* $\mathrm{a}$ is present in the character vector $\mathrm{x}$

$\mathrm{R}$ base package

any unigram, bigram, or trigram of the character vector $\mathrm{a}^{* *}$ is present in the text

any element of the character vector a is followed by any element of the character vector $b$ in the text, in the same part of the sentence, and within the radius provided.

\section{- If number $=1$}

any element of the character vector a is present in the text, but is not followed by any element of the character vector $b$ within the radius provided,

- If number=2

any element of the character vector b is present in the text, but is not preceded by any element of the character vector a within the radius provided,

- If number $=3$

any element of the character vector b is present in the text, but is not preceded by any element of the character vector a within the radius provided, nor followed by any element of the character vector $\mathrm{c}$ within the radius provided any element of the character vector $\mathrm{c}$ is not sandwiched between any element of the character vector a and any element of the character vector $b$

sandwich.wrap(a, b, c, text)

*the term "character vector" simply refers to an ordered vector of elements. The character vectors $a, b$, and $c$, are used to represent the content of specific and generic keywords dictionaries. The order in which the elements appear in the dictionaries does not matter. On the other hand, the character vector $x$ is the text of the injury report split based on whitespace (last preprocessing step previously described), so the order matters and corresponds to the order in which elements appear in the text.

** in this case, the elements of $a$ have to be regular expressions 
The functions summarized in Table 4 offer the same kind of flexibility than the NEAR, AFTER, BEFORE, OR, AND, and NOT functions proposed in the commercial content analysis software Wordstat 6.0 (Provalis research 2014). In addition, rules are limited to a maximum of 2 function-based statements in Wordstat. Of course, Rbased rules are not limited in their number of statements, so variables can be detected based on more intricate patterns, and finer tuning can be achieved. Also, in Wordstat, the items passed to each function can only be picked from a limited number of pre-defined categories. The $\mathrm{R}$ functions developed for this research can be used for any combination of elements (unigrams, bigrams, trigrams, punctuation marks, and numbers). These combinations can take the form of multiple dictionaries assembled, subsets of dictionaries, and keywords punctually prescribed. There is no limitation in quantity and origin of the elements. These functions are available on request by emailing the corresponding author.

\subsubsection{Illustrative Example}

An example of rule building using some of the $\mathrm{R}$ functions previously introduced will be presented. These examples consider the incident report: "the employee was walking down the stairs and slipped". After being cleaned and structured into an ordered vector of elements, this report can be represented as shown in Figure 3.

$$
\text { employee walking down stairs and slipped }
$$

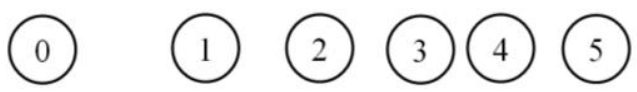

\section{Figure 3. Cleaned and structured incident report}

If the goal is to detect the attribute slippery walking surface, one rule can be written in English as "any element corresponding to the idea of people is followed by any element from the topic slipping within a radius of 7". This rule can be translated in $\mathrm{R}$ as a single complex.s() statement, and is capable of detecting the variable slippery walking surface, as illustrated in Figure 4.

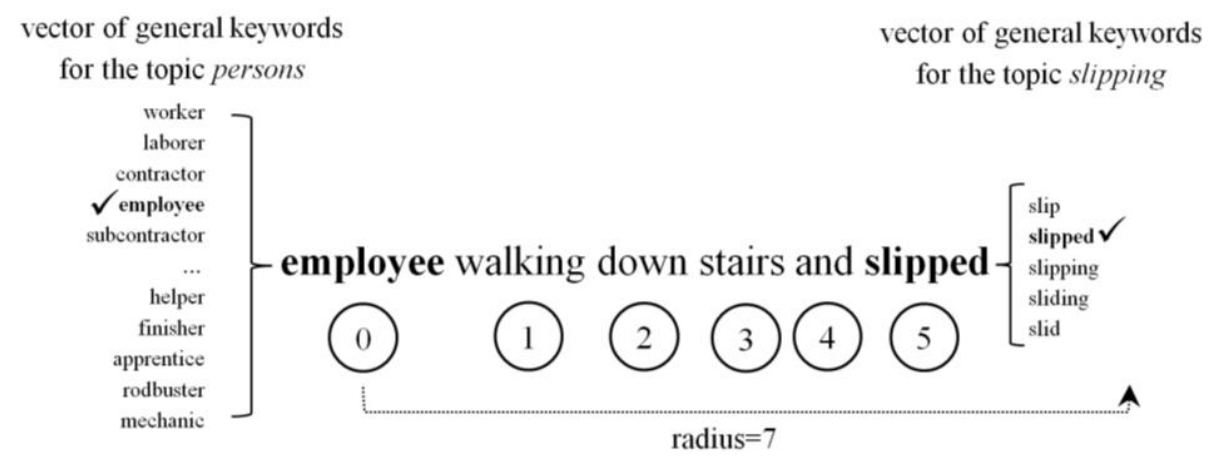

Figure 4. Detection of the variable slippery walking surface based on a single complex.s() statement

However, caution should be used when working with radii. If the distance prescribed is too short, variables can go undetected (a radius of 3 in the example at hand, for example). However, if the distance prescribed is too long, the risk of capturing spurious relationships and thus of improperly detecting variables is increased, as shown in Figure 5 . 


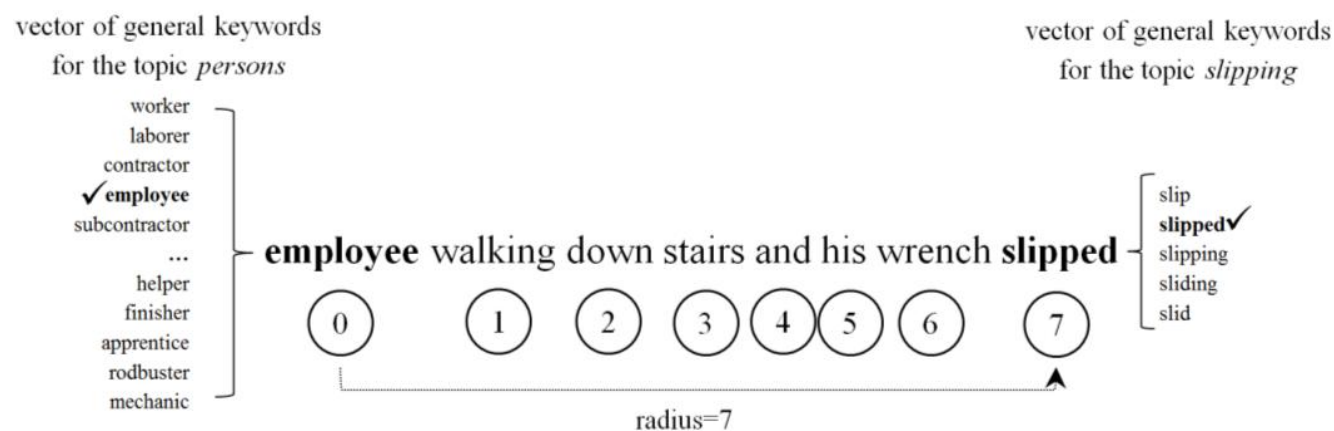

Figure 5. Faulty detection of the variable slippery walking surface by a single complex.s() statement

In order to minimize the risk of such false alarms, the rule can be modified as follows: "(any element corresponding to the idea of people is followed by any element from the topic slipping within a radius of 7) AND NOT (any element referring to the topic slipping is preceded by any element of the topic tools within a radius of 3)". This rule can be written in R using two complex.s() statements, as shown in Figure 6. With such a new rule, the false alarm is avoided.

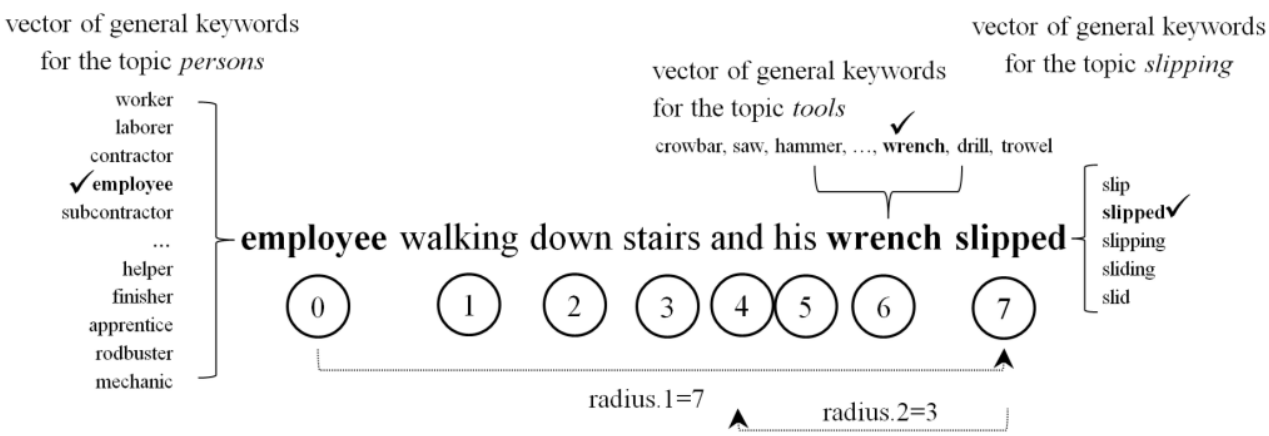

Figure 6. Two complex.s() statements allow the false alarm to be avoided

Furthermore, because only persons and (materials or tools) can slip, the rule can be simplified as: "any element from the topic slipping is present, but this element is not preceded by any element from the topic materials.tools within a radius of 3". This is written in R as a single tricky.double() statement. Some other noteworthy examples of when tricky.double() statements prove useful include "line of fire" (thermal should be detected when "fire" is present but not when "fire" is immediately preceded by "line of"), "chain fall", (a chain fall is a tool and has nothing to do with falling), "concrete vibrator" (concrete should be detected when "concrete" is present but not when it is immediately followed by "vibrator", "hammer", etc.), "rebar foreman" (rebar should be detected when "rebar" is present but not when it is immediately followed by "foreman", "finisher", etc.), "chipping hammer" (chipping should be detected when "chipping" is present, but not when it is immediately followed by "hammer"), or "finger nail", "nail gun", etc. (nail should be detected when "nail" is present, but not when it is immediately preceded by "finger", "thumb", "index", etc., nor followed by "gun", "driver", etc.).

\subsection{Processing incident reports}

When presented with reports, as shown in Figure 1, the NLP tool starts by selecting the first report. The report is cleaned, and scanned for any specific keyword associated with any variable. Then, detection rules for all variables are tested. When all attempts to detect variables have been made, a binary vector of length the total number of variables is returned. The binary vector features "1" whenever the corresponding variables have been detected, and "0" elsewhere. These steps are repeated for all the other reports. Because all reports are scanned in sequence, parallel processing could be used to speed up the process. For that purpose, the "doParallel" R package (Revolution Analytics and Steve Weston 2014) was used. By using 36 cores at 2.6GHz, 4,377 reports could be 
analyzed in just under 11 minutes. After the tool is done looping through all the reports, a binary matrix is obtained, as illustrated by Figure 7. The following step consists in resolving conflicts.

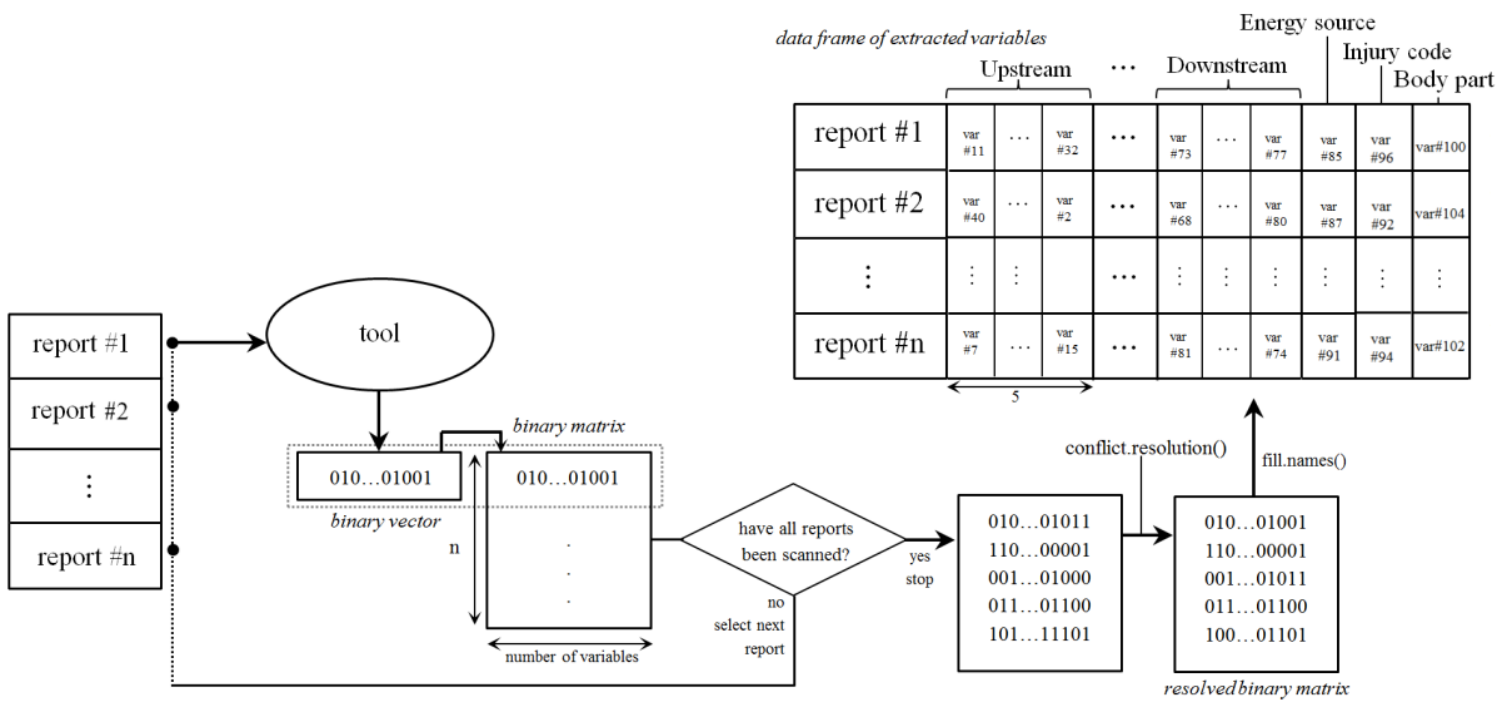

Figure 7. Scanning of injury reports, and post-processing

\subsection{Resolving conflicts among detected variables}

The reasoning behind using a clash detection and resolution function is that some variables are incompatible, such as working at height and working below elevated workspace, while some others are implicitly connected, like radiation and exposure to harmful substance. After a given report has been scanned, it is thus necessary to make sure that the variables that have been detected are not incompatible and that no natural association has been missed.

For example, if the keyword "ice" is present, the attribute ice will be detected (except in cases where ice packs are applied on bruises, or ice buckets are carried, etc.). But because topics about persons and slipping will always be present in ice-related incident reports, and moreover combined in the same fashion ("employee slipped on [...]"), the attribute slippery walking surface will always be detected if ice is detected. While not fundamentally incorrect (ice is indeed a subset of slippery walking surface), this association is problematic. In fact, the attribute-based framework strives to produce high quality structured data on which statistical models can be applied. For that purpose, overlaps in the attributes are to be avoided as much as possible to ensure that every precursor keeps its full predictive power. Therefore, conflict resolution rules were created. In this example, the rule is to delete slippery walking surface whenever ice is present. Examples of similar conflict resolution rules include (but are not limited to) sparks and small particles (sparks is preferred), stairs and ladder (ladder is preferred), fall on same level and fall to lower level (fall to lower level is preferred).

In addition to rules precluding redundancies, other rules were developed to ensure proper association. Indeed, some variables should always be found together. For example the injury code struck by or against should always be associated with the energy source motion. In the same manner, the presence of the energy sources electricity, thermal, or chemical, should always trigger the detection of the injury code exposure to harmful substance. Finally, if the injury codes fall to lower level or fall to same level have been detected, the energy source gravity should always be added. Such rules serve as an internal control in the coding structure, and were integrated into the conflict.resolution() function. 
557 As described in Figure 7, when all conflicts have been resolved by the conflict.resolution() function, the binary 558 matrix is converted to a textual matrix by the fill.names() function. This matrix comprises injury reports as rows, 559 and the attributes and outcomes detected as columns (see Table 5). Both matrices (binary and textual) are 560 automatically written as Excel spreadsheets, and can also be analyzed directly in R. 
Table 5. Example of the system's output for 12 injury reports

\begin{tabular}{|c|c|c|c|c|c|c|c|c|c|c|}
\hline Description & up. 1 & up. 2 & up. 3 & trans.1 & trans. 2 & $\operatorname{trans} .3$ & down.1 & energy & code & body part \\
\hline $\begin{array}{l}\text { Employee was welding on a pipe, as he } \\
\text { brought hands down he touched the } \\
\text { tungsten with left finger resulting in a burn. }\end{array}$ & piping & welding & & & & & & thermal & $\begin{array}{l}\text { exposure to harmful } \\
\text { substance }\end{array}$ & upper extremities \\
\hline $\begin{array}{l}\text { The employee was in the process of } \\
\text { hoisting a piece of cable tray to an above } \\
\text { level and he scraped his arm on a sharp } \\
\text { edge of the cable tray. }\end{array}$ & cable tray & & & $\begin{array}{l}\text { unpowered } \\
\text { tool }\end{array}$ & $\begin{array}{l}\text { lifting } \\
\text { pulling } \\
\text { manual } \\
\text { handling }\end{array}$ & sharp edge & & motion & struck by or against & upper extremities \\
\hline $\begin{array}{l}\text { Climbing out of scaffold and felt back } \\
\text { discomfort. }\end{array}$ & scaffold & $\begin{array}{l}\text { working at } \\
\text { height }\end{array}$ & & exiting & & & & motion & overexertion & trunk \\
\hline $\begin{array}{l}\text { Employee was grinding a pipe in a tight } \\
\text { spot, grinder kicked back making contact } \\
\text { with right thumb resulting in an abrasion. }\end{array}$ & $\begin{array}{l}\text { confined } \\
\text { workspace }\end{array}$ & grinding & piping & powered tool & & & & mechanical & struck by or against & upper extremities \\
\hline $\begin{array}{l}\text { EE was lifting a } 2 \text { X } 12 \text { wood plank when } \\
\text { the wood plank got too heavy causing it to } \\
\text { fall back towards the EE and hit him on the } \\
\text { top/front of his hard hat. }\end{array}$ & $\begin{array}{l}\text { heavy } \\
\text { material/tool }\end{array}$ & lumber & & $\begin{array}{l}\text { lifting pulling } \\
\text { manual } \\
\text { handling }\end{array}$ & & & $\begin{array}{l}\text { improper security } \\
\text { of materials }\end{array}$ & gravity & struck by or against & head \\
\hline Welding FOB & welding & & & small particle & & & & motion & struck by or against & head \\
\hline $\begin{array}{l}\text { Employee was offloading burners with a } \\
\text { cart when the cart moved unexpectedly } \\
\text { "crushing" his leg between the cart and the } \\
\text { existing railing. }\end{array}$ & $\begin{array}{l}\text { unpowered } \\
\text { transporter }\end{array}$ & $\begin{array}{l}\text { guardrail } \\
\text { /handrail }\end{array}$ & & $\begin{array}{l}\text { lifting pulling } \\
\text { manual } \\
\text { handling }\end{array}$ & & & & motion & $\begin{array}{l}\text { caught } \\
\text { in/compressed }\end{array}$ & lower extremities \\
\hline $\begin{array}{l}\text { Employee was grinding overhead, weight } \\
\text { shifted and board he was standing on slid, } \\
\text { causing a "pop" to upper leg resulting in a } \\
\text { strain. }\end{array}$ & grinding & lumber & & & $\begin{array}{l}\text { working } \\
\text { overhead }\end{array}$ & $\begin{array}{l}\text { unstable } \\
\text { support } \\
\text { /surface }\end{array}$ & & motion & overexertion & lower extremities \\
\hline $\begin{array}{l}\text { Laborer suffered concrete burns during a } \\
\text { chipping operation. }\end{array}$ & chipping & & & & & & & chemical & $\begin{array}{l}\text { exposure to harmful } \\
\text { substance }\end{array}$ & not detectable \\
\hline $\begin{array}{l}\text { The employee reported that he received an } \\
\text { insect bite/sting on } 6 / 21 / 13 \text { at work. }\end{array}$ & & & & insect & & & & biological & $\begin{array}{l}\text { exposure to harmful } \\
\text { substance }\end{array}$ & not detectable \\
\hline $\begin{array}{l}\text { Employee was walking out of the } \\
\text { warehouse building. The floor was wet } \\
\text { from the rain. He slipped and fell on his } \\
\text { left knee on the concrete floor. }\end{array}$ & concrete & $\begin{array}{l}\text { slippery } \\
\text { walking } \\
\text { surface }\end{array}$ & exiting & & & & & gravity & struck by or against & lower extremities \\
\hline $\begin{array}{l}\text { An employee of --- Mechanical was using } \\
\text { Oxy/Acetylene to cut a pipe when hot slag }\end{array}$ & piping & welding & & slag & & & $\begin{array}{l}\text { no or improper } \\
\text { PPE }\end{array}$ & thermal & $\begin{array}{l}\text { exposure to harmful } \\
\text { substance }\end{array}$ & upper extremities \\
\hline
\end{tabular}


601

602

603

604

605

\section{VALIDATION OF THE SYSTEM AND MEASURMENT OF RELIABILITY}

As shown in Figure 2, the tool tuning process was iterative. At each step, 140 injury reports were randomly drawn without replacement from the dataset of 2,201 reports of Desvignes (2014) and Prades (2014). These reports were then automatically scanned by the R system, and as previously explained, a textual matrix of 140 rows by 19 columns ( 1 column for the textual reports, 15 for the attributes, 1 for energy source, 1 for injury code, and 1 for body part) similar to Table 5, was returned. This output was divided into seven textual matrices of 20 rows each. Each table was assigned to a researcher, who had been involved with the Desvignes (2014) study and was familiar with the coding scheme and operational definitions of variables. As illustrated by the retrieval matrix in Table 6, each researcher reviewed their randomly assigned piece of output looking for true positives (TP), false positives (FP) and false negatives $(\mathrm{FN})$.

\section{Table 6. Retrieval matrix (adapted from Buckland and Grey 1994).}

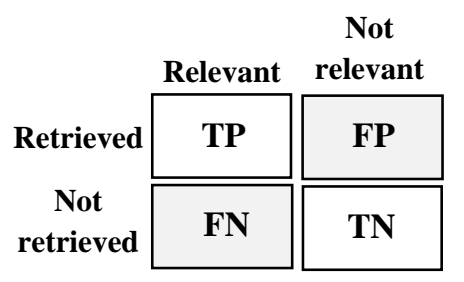

True positives (TP), also informally called "hits", refer to cases when the tool has rightfully detected a precursor that was indeed present in the injury report. False positives (FP), also called "false alarms", or type I error, designate an instance when the tool has wrongfully detected an attribute that was not present in the injury report (not relevant). Finally, false negatives (FN), also called "misses", or type II error, are cases when the tool has omitted to detect the presence of a precursor that was actually there in the report (not detected and relevant). It should be noted that the last option, true negatives (TN), occurs when the tool has not detected an attribute that was not present in the injury report. True negatives were not taken into account.

After careful examination by the seven researchers, the reviewed textual matrices were aggregated and all true positives, false positives, and false negatives were counted. Three performance metrics, standard in the field of information retrieval and NLP, were then computed: precision, recall and F-1 score (Al Qady and Kandil 2014, Yu and Hsu 2013, Buckland and Grey 1994). It should be noted that inspection of the system's output agreed upon by seven humans was preferred over automatic comparison to a gold standard (e.g., manually labeled reports). Indeed, this allowed the source of each error made by the system to be investigated, identified, and fixed by tuning the grammatical rules and lexicon accordingly. This output examination process played a crucial role in developing a highly accurate system.

Precision is calculated, as shown in equation 1, as the proportion of relevant items to the number of items detected (Buckland and Grey 1994). This is simply the probability that an attribute is present given that it was detected by the tool (Goutte and Gaussier 2005). High precision means that most results returned are relevant. Maximum precision is attained in the absence of type I error (i.e., no false positive). On the other hand, recall is the number of retrieved relevant items as a proportion of all relevant items (see equation 2). In other words, recall is the probability that a precursor that is present is detected by the tool. A high recall rate means that most of the relevant results are returned, and recall is maximized in the absence of misses (i.e., in the absence of type II error). Buckland and Grey (1994) define precision as the purity of retrieval and recall as the completeness of retrieval. 


$$
\text { precision }=\frac{\text { true positives }}{\text { true positives }+ \text { false positives }} \quad \text { recall }=\frac{\text { true positives }}{\text { true positives }+ \text { false negatives }}
$$

(Equation1)

(Equation 2)

More precisely, the formulas that were used macro-averaged the results (Prabowo and Thelwall 2009). Instead of considering recall and precision rates for each variable separately (what is known as microaveraging), true positives, false positives, and false negatives for all categories were aggregated and the recall and precision rates averaged these counts. Macro-averaging treats each class equally, and is harsher than micro-averaging since one class that results in a bad performance can significantly deteriorate the overall performance (Prabowo and Thelwall 2009).

Finally, the F-1 score is defined as the harmonic mean of precision and recall. As shown in equation 3, the F-1 score gives the same weight to precision and recall, assuming that the cost of a false positive equals the benefit of a true positive.

$$
F 1 \text { score }=2 \cdot \frac{\text { precision } \cdot \text { recall }}{\text { precision }+ \text { recall }}
$$

(Equation 3)

A threshold of $95 \%$ in F-1 score was selected as a tool tuning stop criterion. This is a high threshold, especially when using strict macro-averaging performance metrics, but as noted by Grimmer and Stewart (2013), it is crucial to show that the automated program is able to replicate human coding. The scores for each round of random reviews are summarized in Table 7. Four iterations were needed before the 95\% threshold in F-1 score was achieved. As shown in Figure 2, lessons learned from careful examination of the errors made by the system played a huge role in improving skill.

The error rates for energy source and injury code, simply defined as the number of errors divided by the number of reports scanned $(n=140)$, were also computed at each round. For body part, to avoid any false alarm, the tool was designed to return not detectable when more than one body part is detected, or when the information is not present in the report. The detection of body part was only added to the system's functionality after the third iteration of random reviews, and not detectable was returned $6.25 \%$ of the time. When any body part was detected, the system was correct every time.

\section{Table 7. System's performance at each step of the tuning process}

$\begin{array}{ccccccc}\text { iteration } & \text { precision } & \text { recall } & \text { F1-score } & \begin{array}{c}\text { error rate for } \\ \text { energy source }\end{array} & \begin{array}{c}\text { error rate for } \\ \text { injury code }\end{array} & \begin{array}{c}\text { "not detectable" rate } \\ \text { for body part }\end{array} \\ 1 & 0.854 & 0.924 & 0.888 & 11.5 \% & 8.0 \% & \text { NA } \\ 2 & 0.911 & 0.946 & 0.928 & 8.6 \% & 4.3 \% & \text { NA } \\ 3 & 0.917 & 0.959 & 0.938 & 3.6 \% & 2.9 \% & \text { NA } \\ 4 & 0.950 & 0.970 & 0.960 & 5.7 \% & 5.7 \% & 6.25 \%\end{array}$

These scores are comparable or even better than the scores attained by most statistical classifiers found in the literature. For instance, Verma et al. (2011) used a naïve Bayes classifier to analyze tweets and reached $80 \%$ accuracy. Tweets were to be classified into 5 categories. A final accuracy of 0.65 with a recall rate of 0.75 was obtained by Grimmer and Stewart (2013), with a random forest classifier. Go et al. (2009) used unigrams and bigrams as variables to classify tweets as positive or negatives and an accuracy of $83 \%$ was reached. Finally, Bai (2011) reviewed two studies about automated opinion mining and movie review polarity categorization and reported that the accuracies ranged between $66 \%$ and $88.9 \%$. 
664

665

666

667

668

669

670

671

672

673

674

675

676

677

678

679

680

681

682

683

684

685

686

687

688

In the construction field specifically, Al Qady and Kandil (2014) implemented unsupervised clustering algorithms to classify project documents into mutually exclusive classes and reached a $0.844 \mathrm{~F}$-score in the optimum case. The computer aided drawings automated retrieval system of $\mathrm{Yu}$ and Hsu (2013) attained $100 \%$ recall, but the precision was only $57.2 \%$. Finally, $86.37 \%$ accuracy was reached by Caldas and Soibelman (2003), who used Support Vector Machines to classify construction project documents into hierarchical classes.

It is not surprising that the system based on handcrafted rules developed in this research outperformed all the aforementioned machine learning based tools, since as noted by Sagae and Lavie (2003), hand coded rules allow researchers to transfer their expertise, knowledge of the data, and human intelligence to the system. Tools based on manually devised rules are usually capable of deeper understanding than machine learning classifiers when the domain of application is very specific (Wang et al. 2002).

\section{CONCLUSION, LIMITATIONS, AND RECOMMENDATIONS}

Major construction firms and federal agencies have been recording injury-related events and near misses in the form of digital textual reports for many years, but due to the lack of an adapted framework and methodology, and due to the high time, labor, and organizational costs related to manual content analysis (not to mention inter-coder reliability issues), the most part of this valuable knowledge is left unstructured and unexploited. Specifically, low severity, high frequency events that are not OSHA-recordable but account for huge financial and long term human costs are very seldom studied.

This research tested the proposition that the needs for manual content analysis of incident reports can be eliminated using NLP. Results clearly show that this is possible. The R system that was developed in this research is capable of scanning naturally occurring, unstructured textual injury reports for 101 relevant, valid and carefully defined variables ( 80 precursors, 7 injury types, 9 energy sources, and 5 body parts) with high recall (0.97) and precision (0.95) rates. The keyword dictionaries and all $\mathrm{R}$ functions developed are available on request by emailing the corresponding author. Some functions are also provided in the appendix. As will be discussed in the recommendations section, the proposed NLP system will enable organizations to quickly and automatically extract the knowledge contained in their databases of unstructured injury reports to improve safety management.

The attribute-based framework of Esmaeili and Hallowell (2012), refined by Prades (2014) and Desvignes (2014) showed great promise with respect to extracting useful and standardized information from unstructured injury reports. However, the large-scale use of this framework was previously limited by the high costs and issues related to manual content analysis. Consequently, all the subsequent analyses necessary to identify trends and patterns in the attribute and outcome data (i.e., extract insight), were limited by the small amount of data available. The tool developed in this study removes the need for manual content analysis of injury reports, enabling very high numbers of reports to be scanned for attributes and outcomes, statistical modeling to be applied on very large data sets, and more insight to be extracted.

\subsection{Limitations}

First, the system is inherently limited by the use of hard detection rules: it is not robust to unfamiliar and erroneous input, such as misspelled, missing, and unseen words. In other words, the system cannot address situations that were not anticipated, and the quality of the available textual data directly affects the quality of the attribute and outcome data extracted by the system. For instance, when faced with a misspelled word (e.g., "steal" instead of "steel") the R system is unable to detect the associated variable steel/steel sections. Most frequent misspellings can be anticipated, but obviously, it is unfeasible to account for all possible cases. Also, some reports contain a description of the events following the incident (e.g., "[...] worker was brought to the job trailer and an ointment was applied"). Despite the 
many precautions that were taken, precursors can still be wrongfully detected from these irrelevant portions of the text.

The R system reached very high scores during random reviews of its output, indicating that the impact of erroneous or misleading input was very limited. Indeed, the reports available to this research were generally carefully written, and only contained facts relevant to the incident. However, verifying the quality of the injury reports is a necessary first step that should always be taken before running the R tool on any new database in the future. Reports of overly poor quality may prevent the automated content analysis from being successfully conducted.

The methodology introduced in this study is applicable to other domains. Especially, the system structure (i.e., the overall approach, methodology and rule-writing functions that were developed) is ready to be used in any situation. Also, the full system as it is is ready to scan incident reports pertaining to the industrial, energy, infrastructure, and mining fields, since the grammatical and conflict resolution rules as well as the lexicon developed in this research, were tuned and validated on such reports. If scanning reports belonging to other industry sectors is wanted, a prerequisite for reaching high skill will be to extend the tool's dictionaries and detection rules. Defining new fundamental attributes may also be needed, which requires trained content analysts and calibration meetings. As Grimmer and Stewart (2013) note, a limitation of dictionary-based methods is that they are only efficient inside the domain for which the dictionaries were originally developed.

Finally, using the tool requires installing $\mathrm{R}$ on the machine. Knowledge of $\mathrm{R}$ is not needed, as the process is fully automatic (a single command only has to be executed by the user). However, tuning the tool (i.e., creating/updating rules) requires basic $\mathrm{R}$ literacy.

\subsection{Recommendations for future research}

When a sufficient amount of reports have been classified by the R system in each category, a natural next step would consist in hybridizing the system with machine learning algorithms such as Support Vector Machines. As a hybrid, the system would keep the deep understanding given by hand coded rules, while acquiring the flexibility of statistical classifiers. Moreover, by judiciously aggregating the decisions of hard and soft detection rules, the skill of the system could increase even more (Prabowo and Thelwall 2009).

In order to get closer to $100 \%$ accuracy, the errors made by the tool can be automatically detected using data mining methods such as hierarchical clustering, which is known for its ability to isolate outliers in small clusters. Manually inspecting these small clusters allows easy identification and correction of the errors. This approach can be used for instance to attain a maximal signal over noise ratio before training statistical predictive models on the data.

The main contribution of the proposed NLP system is its ability to extract meaningful structured attribute and outcome data from unstructured injury reports automatically and with high accuracy. Such structured data represents raw material required to apply data mining and statistical modeling algorithms that will allow to better understand, predict, and prevent the occurrence of construction accidents. For instance, models predicting safety outcomes from combinations of attributes will assist safety managers in accurately diagnosing the safety risk associated with specific construction situations and provide them with tailored recommendations based on simple observations of the work environment. The proposed system can be seen as a construction safety knowledge discovery tool: large databases of injury reports represent a wealth of valuable lessons waiting to be learned and made readily available to construction professionals in order to help decision-making and to prevent mistakes from being repeated over and over. 
The methodology presented in this research can be applied to mine other construction textual data like contracts and project documentation, but more generally, it can be applied to mine any kind of text. The use of NLP may soon become mandatory and widespread in construction management to make sense of the ever-growing amount of digital information associated today with even the smallest construction project. Statistical classifiers and machine learning algorithms are without a doubt the present and the future of text analytics, but this study shows that when very high accuracy is sought on a specific, welldefined domain, dictionaries and hand-coded rules can bring better results, once a one-time investment has been made.

\section{ACKNOWLEDGEMENTS}

We would like to thank the National Science Foundation for supporting this research through an Early Career Award (CAREER) Program. This material is based upon work supported by the National Science Foundation under Grant No. 1253179. Any opinions, findings, and conclusions or recommendations expressed in this material are those of the authors and do not necessarily reflect the views of the National Science Foundation. We would also like to recognize Bentley Systems for their financial support for this research and intellectual contributions.

\section{REFERENCES}

Adrian A. Dragulescu (2013). "xlsx: Read, write, format Excel 2007 and Excel 97/2000/XP/2003 files." $\mathrm{R}$ package version 0.5 .5 . http://CRAN.R-project.org/package $=\mathrm{xlsx}$

Al Qady, M., \& Kandil, A. (2014). Automatic clustering of construction project documents based on textual similarity. Automation in Construction, 42, 36-49.

Albert, A., Hallowell, M. R., Kleiner, B., Chen, A., \& Golparvar-Fard, M. (2014). Enhancing construction hazard recognition with high-fidelity augmented virtuality. Journal of Construction Engineering and Management, 140(7).

Pang, B., Lee, L., \& Vaithyanathan, S. (2002, July). Thumbs up?: sentiment classification using machine learning techniques. In Proceedings of the ACL-02 conference on Empirical methods in natural language processing-Volume 10 (pp. 79-86). Association for Computational Linguistics.

Bai, X. (2011). Predicting consumer sentiments from online text. Decision Support Systems, 50(4), 732742.

Baradan, S., \& Usmen, M. A. (2006). Comparative injury and fatality risk analysis of building trades. Journal of construction engineering and management, 132(5), 533-539.

Barbella, D., Benzaid, S., Christensen, J. M., Jackson, B., Qin, X. V., \& Musicant, D. R. (2009, July). Understanding Support Vector Machine Classifications via a Recommender System-Like Approach. In DMIN (pp. 305-311).

790

791

792

793

794

795

796

797

798
Beleites, C., Neugebauer, U., Bocklitz, T., Krafft, C., \& Popp, J. (2013). Sample size planning for classification models. Analytica chimica acta, 760, 25-33.

Bishop, C. M. (1995). Neural networks for pattern recognition.

Breiman, L. (2001). Random forests. Machine learning, 45(1), 5-32. 
Buckland, M. K., \& Gey, F. C. (1994). The relationship between recall and precision. JASIS, 45(1), 1219.

Caldas, C. H., \& Soibelman, L. (2003). Automating hierarchical document classification for construction management information systems. Automation in Construction, 12(4), 395-406.

Chawla, N. V. (2005). Data mining for imbalanced datasets: An overview. In Data mining and knowledge discovery handbook (pp. 853-867). Springer US.

Chi, N. W., Lin, K. Y., \& Hsieh, S. H. (2014). Using ontology-based text classification to assist Job Hazard Analysis. Advanced Engineering Informatics, 28(4), 381-394.

Chowdhury, G. G. (2003). Natural language processing. Annual review of information science and technology, 37(1), 51-89.

Collins, F. S., Green, E. D., Guttmacher, A. E., \& Guyer, M. S. (2003). A vision for the future of genomics research. Nature, 422(6934), 835-847.

CPWR - The Center for Construction Research and Training, produced with support from the National Institute for Occupational Safety and Health grant number OH009762, 2013

Desvignes, M. "Requisite empirical risk data for integration of safety with advanced technologies and intelligent systems." Masters Thesis, University of Colorado at Boulder, 2014. doi: $10.1061 / 9780784412329.030$

Esmaeili, B. and Hallowell, M. (2012) Attribute-Based Risk Model for Measuring Safety Risk of StruckBy Accidents. Construction Research Congress 2012: pp. 289-298.

doi: $10.1061 / 9780784412329.030$

Esmaeili, B., \& Hallowell, M. R. (2011). Diffusion of safety innovations in the construction industry. Journal of Construction Engineering and Management, 138(8), 955-963.

Esmaeili, Behzad. "Identifying and quantifying construction safety risks at the attribute level." $\mathrm{PhD}$ Dissertation, University of Colorado at Boulder, 2012.

Fisher, I. E., Garnsey, M. R., Goel, S., \& Tam, K. (2010). The role of text analytics and information retrieval in the accounting domain. Journal of Emerging Technologies in Accounting, 7(1), 1-24.

Fleming, M. A. (2009). Hazard recognition. By Design, ASSE, 11-15.

Francis, L., \& Flynn, M. (2010). Text mining handbook. In Casualty Actuarial Society E-Forum, Spring 2010 (Vol. 1).

Go, A., Bhayani, R., \& Huang, L. (2009). Twitter sentiment classification using distant supervision. CS224N Project Report, Stanford, 1-12.

Goutte, C., \& Gaussier, E. (2005). A probabilistic interpretation of precision, recall and F-score, with implication for evaluation. In Advances in information retrieval (pp. 345-359). Springer Berlin Heidelberg. 
Grimmer, J., \& Stewart, B. M. (2013). Text as data: The promise and pitfalls of automatic content analysis methods for political texts. Political Analysis, mps028.

Haddon, W. (1973). Energy damage and the ten countermeasure strategies. Human Factors: The Journal of the Human Factors and Ergonomics Society, 15(4), 355-366.

Hadley Wickham (2012). "stringr: Make it easier to work with strings." R package version 0.6.2. http://CRAN.R-project.org/package=string

Hallowell, M. R. (2008). A formal model for construction safety and health risk management. ProQuest.

Helander, M. G. (1991). Safety hazards and motivation for safe work in the construction industry. International Journal of Industrial Ergonomics, 8(3), 205-223.

Hindle, D. (1989, June). Acquiring disambiguation rules from text. In Proceedings of the 27th annual meeting on Association for Computational Linguistics (pp. 118-125). Association for Computational Linguistics.

Hopkins, D. J., \& King, G. (2010). A method of automated nonparametric content analysis for social science. American Journal of Political Science, 54(1), 229-247.

Hsu, J. Y. (2013). Content-based text mining technique for retrieval of CAD documents. Automation in Construction, 31, 65-74.

Iacobucci, Dawn (ed.) (2001), Journal of Consumer Psychology's Special issue on Methodological and Statistical Concerns of the Experimental Behavioral Researcher, 10 (1\&2), Mahwah, NJ: Lawrence Erlbaum Associates, 71-73

Ingo Feinerer, Kurt Hornik, and David Meyer (2008). "Text Mining Infrastructure in R." Journal of Statistical Software 25(5): 1-54. URL: http://www.jstatsoft.org/v25/i05/.

Joachims, T. (2002). Learning to classify text using support vector machines: Methods, theory and algorithms (p. 205). Kluwer Academic Publishers.

Jung, J., \& Thon, M. R. (2006). Automatic annotation of protein functional class from sparse and imbalanced data sets. In Data Mining and Bioinformatics (pp. 65-77). Springer Berlin Heidelberg.

Karatzoglou, A., \& Feinerer, I. (2010). Kernel-based machine learning for fast text mining in R. Computational Statistics \& Data Analysis, 54(2), 290-297.

Kuechler, W. L. (2007). Business applications of unstructured text. Communications of the ACM, 50(10), 86-93.

Li, N., \& Wu, D. D. (2010). Using text mining and sentiment analysis for online forums hotspot detection and forecast. Decision Support Systems, 48(2), 354-368.

Liddy, E. D. (2001). Natural language processing.

Lombard, M., Snyder-Duch, J., \& Bracken, C. C. (2002). Content analysis in mass communication: Assessment and reporting of intercoder reliability. Human communication research, 28(4), 587-604. 
934

935

936

937

938

939

940

941

942

943

944

945

946

947

948

Manning, C. D. (1999). Foundations of statistical natural language processing. H. Schütze (Ed.). MIT press.

“OSHA Safety and Health Topics.” Accessed March 10, 2015. https://www.osha.gov/SLTC/.

Pereira, F. C., Rodrigues, F., \& Ben-Akiva, M. (2013). Text analysis in incident duration prediction. Transportation Research Part C: Emerging Technologies, 37, 177-192.

Prabowo, R., \& Thelwall, M. (2009). Sentiment analysis: A combined approach. Journal of Informetrics, 3(2), 143-157.

Prades, M. "Attribute-based risk model for assessing risk to industrial construction tasks." Masters Thesis, University of Colorado at Boulder, 2014.

R Core Team (2014). R: A language and environment for statistical computing. R Foundation for Statistical Computing, Vienna, Austria. URL http://www.R-project.org/.

Revolution Analytics and Steve Weston (2014). "doParallel: Foreach parallel adaptor for the parallel package.” R package version 1.0.8. http://CRAN.R-project.org/package=doParallel

Sacks, R., Rozenfeld, O., \& Rosenfeld, Y. (2009). Spatial and temporal exposure to safety hazards in construction. Journal of construction engineering and management, 135(8), 726-736.

Sagae, K., \& Lavie, A. (2003). Combining Rule-based and Data-driven Techniques for Grammatical Relation Extraction in Spoken Language. In Proceedings of the Eighth International Workshop in Parsing Technologies. Nancy, France.

Soibelman, L., Wu, J., Caldas, C., Brilakis, I., \& Lin, K. Y. (2008). Management and analysis of unstructured construction data types. Advanced Engineering Informatics, 22(1), 15-27.

Venables, W. N., and B. D. Ripley. 2002. Modern applied statistics with S. 4th ed. New York: Springer.

Verma, S., Vieweg, S., Corvey, W. J., Palen, L., Martin, J. H., Palmer, M., ... \& Anderson, K. M. (2011, July). Natural Language Processing to the Rescue? Extracting" Situational Awareness" Tweets During Mass Emergency. In ICWSM.

Wang, Y. Y., Acero, A., Chelba, C., Frey, B. J., \& Wong, L. (2002, September). Combination of statistical and rule-based approaches for spoken language understanding. In INTERSPEECH.

Wang, H. H., \& Boukamp, F. (2011). Ontology-based representation and reasoning framework for supporting job hazard analysis. Journal of Computing in Civil Engineering, 25(6), 442-456.

Wei, W. W. S. (1994). Time series analysis. Addison-Wesley publ.

Yeung, C. L., Cheung, C. F., Wang, W. M., \& Tsui, E. (2014). A knowledge extraction and representation system for narrative analysis in the construction industry. Expert Systems with Applications, 41(13), 5710-5722.

Zienkiewics, O. C. (1971). The finite element method in engineering science. McGraw-Hill, Londres. 